\title{
Tracing fine-grained sediment transport around Tokyo Bay using cesium-134 and cesium-137 originating from Fukushima Daiichi Power Plant
}

\author{
Y. Koibuchi \\ Graduate School of Frontier Sciences, The University of Tokyo, Japan
}

\begin{abstract}
Coastal sediment transport, which consists of suspended-load and bed-load, has not been fully understood, since bed-load transport of cohesive sand is difficult to observe. The impact on the total amount of fine-grained cohesive sediment has not been elucidated. Cesium-134 and cesium-137 were spread from the Fukushima Daiichi Nuclear Power Plant (FDNPP) after the earthquake of March 11 of 2011, and attached to the fine-grained sand on the land. The contaminated sand flowed into the coast through the rivers possibly due to the complex physical processes in coastal areas. To quantify the fine-grained sediment transport around Tokyo Bay and inside the bay, field observation was carried out by radionuclide originated from FDNPP as an effective tracer. We successfully quantified the cohesive sediment transport in Tokyo Bay, and found that the cohesive sediment transport deposited in the estuary is greatly dependent on the land use, geometry, river discharge and salinity. In addition, the transport driven by the rainfall was minute, and its behavior was quite different from suspended solids. Although further field observations of radionuclide are necessary, it is clear that fine-grained sediment in the bay from rivers already settled on the river mouth by aggregation. The settled sand will not move even in rainfall events. Also, the transport of radionuclide to the Pacific Ocean may not occur.

Keywords: sediment transport, cesium, bedload, suspended load, Fukushima Daiichi Nuclear Power Plant, Tokyo Bay.
\end{abstract}




\section{Introduction}

Drift sand in estuaries, consisting of suspended sediment and bedload, is very important for understanding coastal geomorphology. Unlike that of suspended sediment, it is difficult to observe the movement of bedload [1]. The factors for this phenomenon such as the relationship between the total amount of bedload and drift sand, and the spatial and temporal variations of bedload flux are not yet fully understood, which complicates the quantitative evaluation of suspended sediment. Furthermore, among the bedload transportation processes, the study of the fine-grained sand is notably delayed due to its cohesiveness. On March 11, 2011, the Fukushima Daiichi Nuclear Power Plant (FDNPP) suffered catastrophic damage during the Great Tohoku Earthquake and in the tsunami that followed. The cooling system was disabled due to power loss. This led to the meltdown of the nuclear fuel rods, which damaged the containment vessel and caused a hydrogen explosion; as a result, radioactive materials were released into the environment [2]. Radioactive materials from the FDNPP spread through the atmosphere as plumes. The largest portion of the radioactive materials released comprised iodine and cesium, as these elements vaporize easily [3, 4]. The halflife of iodine is short (ca. one week), however the half-life of cesium is longer (two years for cesium-134 and 30 years for cesium-137). Because it remains in nature for comparatively longer, cesium is the main concern among the radioactive materials released by this accident. As it falls with rain, cesium forms positive ions, adheres to sediments (negative ions), becomes trapped between clay layers, and goes through specific adsorption [5]. Following that, the finegrained sediment adhered with cesium is carried to estuaries via rivers and then to coastal areas as suspended sediment and bedload [6]. The amount of cesium dissolved in the fine-grained sediment is miniscule and does not need to be considered, i.e., once cesium is adhered to the sediment, it tends to remain there. In this study, the cesium released by the accident is assumed as a tracer of finegrained sediment movement. Then field observations were performed to track the movement of the fine-grained sediment in and around Tokyo Bay by using the radioactive materials emitted from the FDNPP.

\section{Material and method}

\subsection{Sampling Site}

Figure 1 shows the study area, i.e., Tokyo Bay, which is ca. $200 \mathrm{~km}$ from the FDNPP and is surrounded by densely populated urban areas including Metropolitan Tokyo, Kanagawa Prefecture, and Chiba Prefecture [7-9]. After the nuclear power plant accident on March 11, 2011, radioactive materials containing cesium fell with rain. Cesium adhered to the road dust, and the finegrained sediment was transported to Tokyo Bay through precipitation events [10-12]. This area, which is far from Fukushima, was chosen for the following reasons. First, at Fukushima, it was not possible to distinguish between the radioactive materials directly supplied by air and those arriving at the monitoring 
sites by sediment movement. Second, as 70\% of Fukushima is forest, the radioactive materials adhered to the fine-grained sediment are unlikely to travel. On the other hand, the urban areas of Tokyo are paved with asphalt, speeding up the transportation of cesium-containing, fine-grained sediment to the water front. Also, since the fallout of radioactive materials in the Tokyo area was low, the only cause of the increase in radioactive material concentration is the transportation of fine-grained sediments. Also, compared to the Fukushima coastline, which is open to the Pacific Ocean, Tokyo Bay is much less affected by ocean currents and waves, thus making it easier to track the sediment transportation process. The first round of sampling was conducted in October 2011 and covered 200 sites along the Arakawa and Edogawa Rivers that flow into Tokyo Bay. At all these locations, samples of water and sediment were collected, and water temperature, salinity, and turbidity were measured using a multiparameter water quality meter (6600V2; YSI Inc.). After establishing the baseline distribution of radioactive materials, sampling was repeated at the same sites: every three months at 10 locations from $3 \mathrm{~km}$ downstream to $10 \mathrm{~km}$ upstream measured from the Arakawa river mouth, every three months at 8 locations from $3 \mathrm{~km}$ downstream to $15 \mathrm{~km}$ upstream measured from the Edogawa river mouth, and every month at five locations in Tokyo Bay. These samples were promptly brought to the laboratory and analyzed as follows.
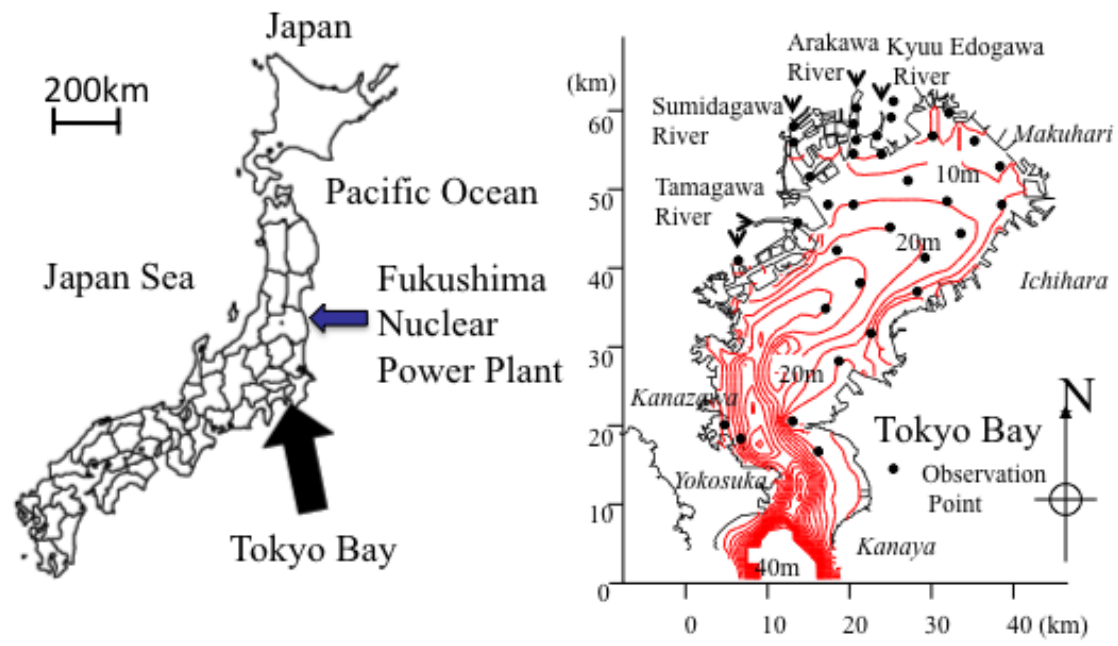

Figure 1: $\quad$ Map of Japan (left) and bathymetry of Tokyo Bay, Japan and its adjacent coastal area, showing the location of the measurement site in the bay (right).

\subsection{Analytical methods}

The bottom sediment samples were fully dried over two hours at $105^{\circ} \mathrm{C}$, and were analyzed using gamma-ray spectrometry and a Germanium (Ge) 
semiconductor detector (GMX; ORTEC Inc.). The sample was placed in an iron and lead chamber to reduce the influence of natural radiation. The apparatus was calibrated using the IAEA-444 soil certified reference material. The particle size of the bottom sediments was measured using a laser diffraction particle size analyzer (SALD-2201; Shimazu Inc.).

The size of the aquatic particles was measured using a Coulter counter (Multisizer3 Coulter Counter; Beckman Coulter Inc.). In addition, aquatic suspended solids of ca. $10 \mathrm{l}$ were filtered and dried using a GFC filter, and the radioactive material in the filter was analyzed using the germanium semiconductor detector.

\section{Results}

\subsection{Cs distribution in Tokyo Bay}

Figure 2 shows the spatial distribution of Cs-134 in the top $1 \mathrm{~cm}$ of the marine surface sediment in Tokyo Bay and along the major rivers flowing into the bay. The figure shows that, as of October 2011, the concentration of Cs-134 was below the detection limit at the center of Tokyo Bay and was above the detection limit at the river estuaries around the bay and at the harbor facilities. Because the ratio of Cs-137 to Cs-134 was similar in these locations, it was inferred that this cesium was of Fukushima origin. This indicates that the cesium was transported with fine-grained sediments along the rivers to Tokyo Bay, and atmospheric input is not a dominant source. Therefore, cesium was used as a tracer of the movement of the fine-grained sediment.

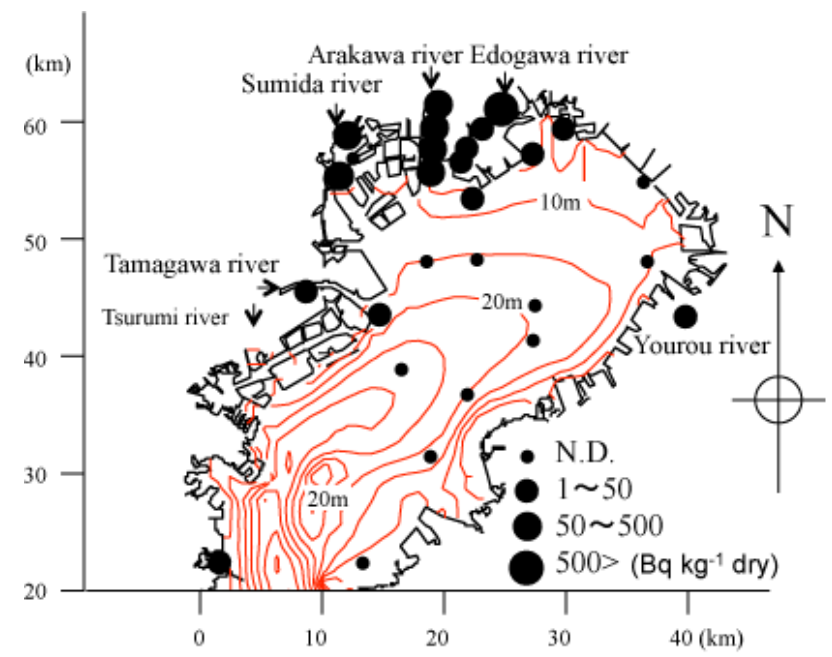

Figure 2: Spatial distribution of Cs-134 in the top $1 \mathrm{~cm}$ of the surface sediment in Tokyo Bay and along the major rivers flowing into the bay of October 2011 . 


\subsection{Cs distribution in the estuaries}

Because cesium was detected only at the estuaries, routine samplings were conducted to examine the spatial distribution of cesium in more detail. Figure 3 shows the spatial distribution of cesium in the top $1 \mathrm{~cm}$ of the bed surface layer in the Edogawa River estuary in October 2011. The horizontal axis represents the distance from the river mouth; the polarity is positive in the upstream direction at $0 \mathrm{~km}$ from the river mouth. The figure shows a large cesium peak at ca. $10 \mathrm{~km}$ upstream from the river mouth. The fine-grained sediment, with cesium adhered, was transported as suspended sediment, then aggregated, and finally deposited in this area. Similarly, Figure 4 shows the spatial distribution of cesium in the top $1 \mathrm{~cm}$ bed surface layer in the Arakawa River estuary. In the Arakawa River, the

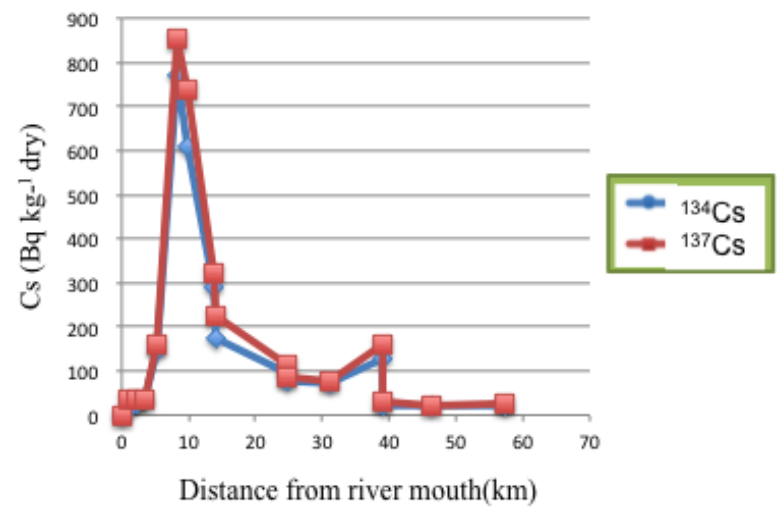

Figure 3: Spatial distribution of cesium in the top $1 \mathrm{~cm}$ of the bed surface layer in the Edogawa River estuary in October 2011.

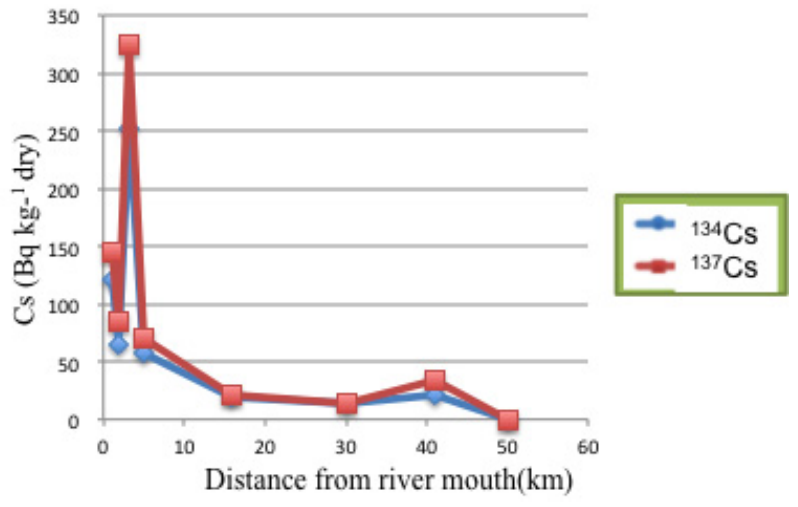

Figure 4: Spatial distribution of cesium in the top $1 \mathrm{~cm}$ bed surface layer in the Arakawa River estuary in October 2011. 
cesium peak is ca. $3 \mathrm{~km}$ downstream from the river mouth but is not as clear as in the Edogawa River. Cesium concentration negatively correlated with particle size, which indicated that cesium was present in the fine-grained sediment.

\subsection{Temporal variation of $\mathrm{Cs}$ in the estuary}

Figure 5 shows the temporal variation of cesium in the Edogawa estuary. The figure shows that the cesium peak gradually decreases with time. Downstream movement is considered to be less since the peak position does not change. The decrease in the cesium concentration may be due to cesium mixing with uncontaminated sediments, or with late-arriving bottom sediment with lower levels of contamination. Another cause may be that cesium is being buried under the near cesium-free sediment arriving later. Similarly, Figure 6 shows the temporal variation of cesium in the Arakawa estuary. Unlike the Edogawa River, the peak, located ca. $3 \mathrm{~km}$ upstream in December 2011, moved downstream with time. The fine-grained sediment was transported to the direction of the river mouth and reached it in September 2012. This indicates that the once deposited, fine-grained sediment was later transported as bedload.

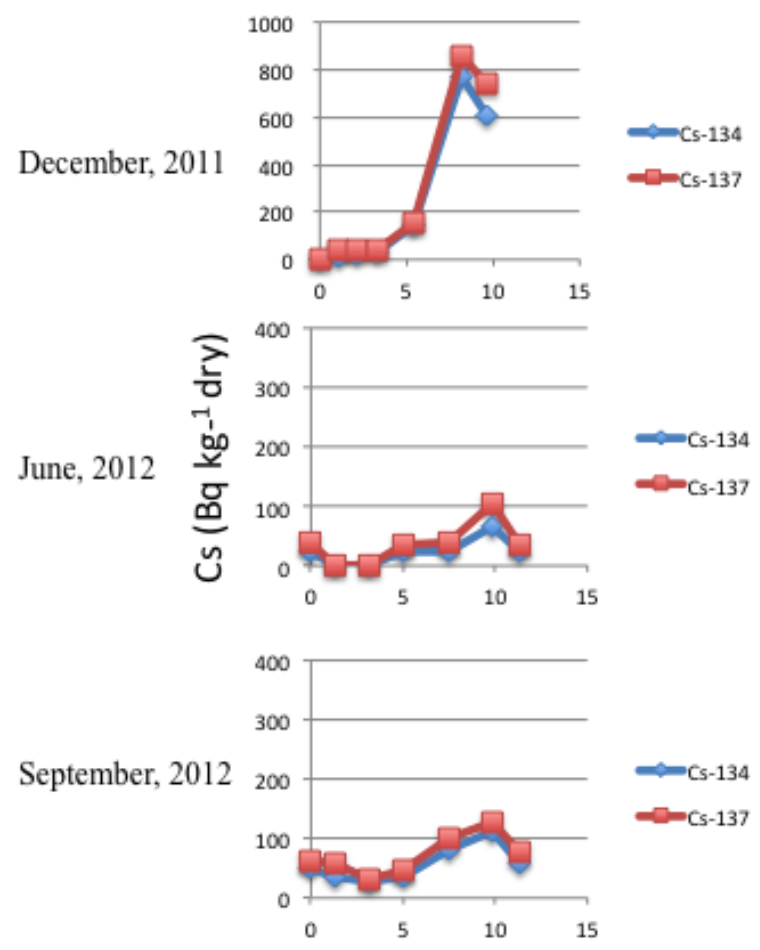

\section{Distance from river mouth $(\mathrm{km})$}

Figure 5: Temporal variation of cesium in the Edogawa. 


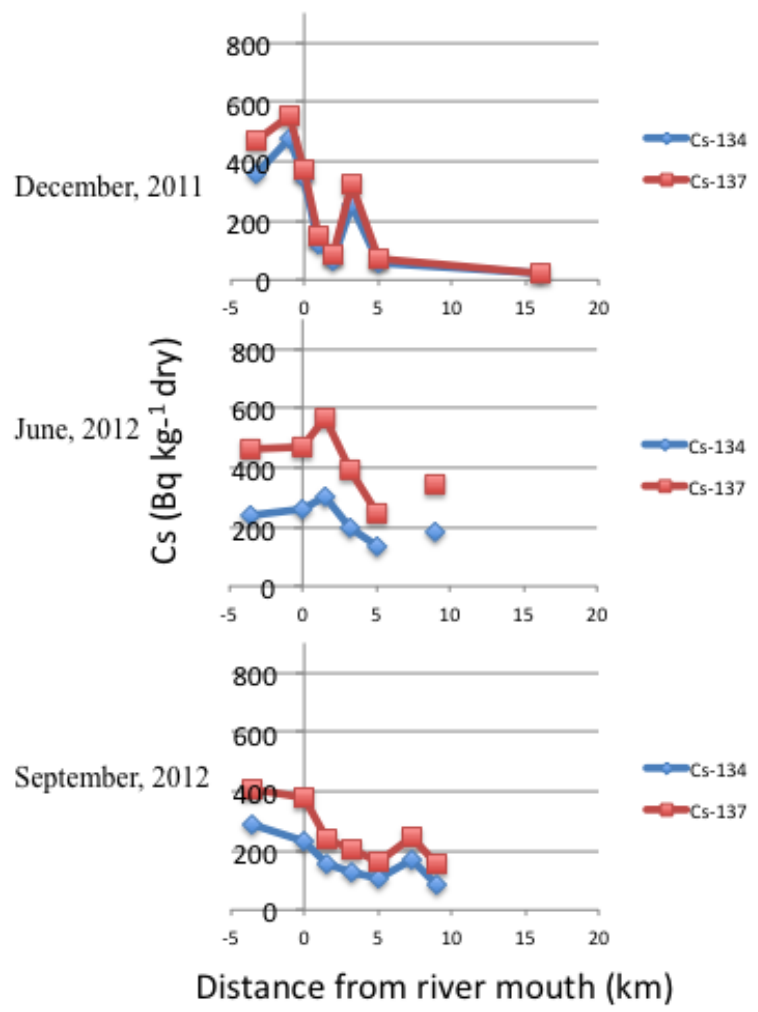

Figure 6: Temporal variation of cesium in the Arakawa estuary.

\subsection{Cesium distribution in the sediment}

In both the Edogawa and Arakawa Rivers, cesium was concentrated and deposited near the estuary, though the exact distance from the river mouth is not the same. In contrast, the cesium transportation trend was different. From the concentration data for the riverbed, it cannot be determined if the decrease is due to either mixing or a newly-deposited sediment layer. Therefore, the vertical distribution was also examined in the sediment core. Figure 7 shows the vertical distribution of cesium in the sediment both at the river mouth of the Edogawa River and at $10 \mathrm{~km}$ from the river mouth. Zero refers to the surface layer of the riverbed. Both at the river mouth and $10 \mathrm{~km}$ upstream, a peak is found at ca. $14 \mathrm{~cm}$ below the riverbed surface. Figure 8 similarly shows the vertical distribution of cesium in the sediment in the Arakawa River. The figure indicates that there is a peak at the surface layer of the river mouth and at $10 \mathrm{~cm}$ below the riverbed surface at ca. $10 \mathrm{~km}$ upstream. 


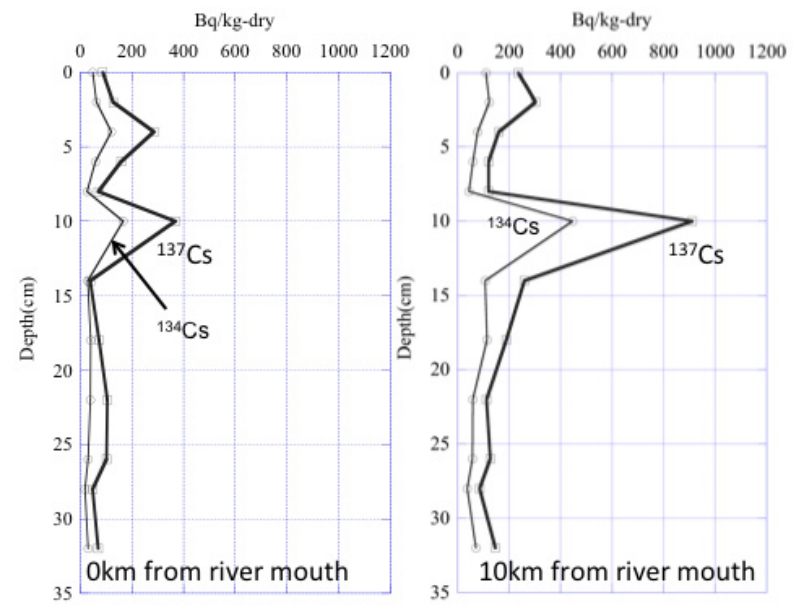

Figure 7: Vertical distribution of cesium in the sediment at the river mouth of the Edogawa River (left) and at $10 \mathrm{~km}$ from the river mouth (right).
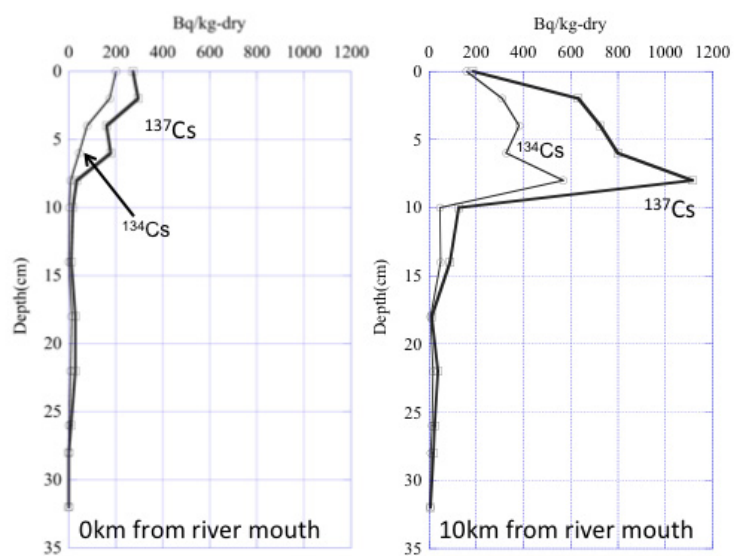

Figure 8: Vertical distribution of cesium in the sediment both at the river mouth of the Arakawa River (left) and at $10 \mathrm{~km}$ from the river mouth (right). 


\section{Discussion}

\subsection{Movement of bedload in the estuary}

Adsorbed to the soil and road dust, cesium is transported by rain during poor weather conditions such as storms. Therefore, its transportation process is based on events. Similarly, the fine-grained sediment containing cesium is washed away and moves downstream when the river flow is large, making it also eventbased. This sediment transportation process is enhanced after rainfall because of the sediment re-suspension by large waves. The reachable depth in the vertical direction, which is a benchmark of water mass movement due to wave impact, is ca. $10 \mathrm{~m}$ in the case of waves with a significant wave period of $4 \mathrm{~s}$ observed in Tokyo Bay where deep water waves were taken into account (Nakagawa et al. [13]). Therefore, in areas with shallow water depth and tendency for wave development, the influence of wave forces is immediately felt up to ca. $10 \mathrm{~m}$. The water depth is ca. $5 \mathrm{~m}$ in the Edogawa and Arakawa estuaries, and the radioactive materials will probably be distributed by repeated sedimentation and re-suspension. In the Edogawa River, the peak was $10 \mathrm{~km}$ upstream of the river mouth and tended to decrease with time. Because the peak is $14 \mathrm{~cm}$ below the surface of the sediment core, it may be assumed that the fine-grained sediment deposited last year is being buried under the recent, much less contaminated sediment. It was also found that the downstream flow of bedload is extremely small.

On the other hand, in the Arakawa River, the peak location was observed to move towards the river mouth direction, and the bedload moved at a speed of ca. $3.5 \mathrm{~km} /$ year.

The difference in the bedload movement between the two rivers possibly depends on several factors including river flow and geomorphology. By comparing the Edogawa and Arakawa Rivers, the observed river flow rate is 1.5 times greater in the Arakawa River, making the downstream movement less prominent in the Edogawa River. In addition, the Edogawa River is a distributary of the Tone River, and the upstream junction has a dam to contain the river flow. During large water flow, peak flow volume is cut, thus accelerating sedimentation and decreasing sediment transportation toward the river mouth. As shown above, the space-time variation of cesium delineated the characteristic differences between the two rivers and helped clarify the transport of bedload, which had been difficult to evaluate in the past.

\subsection{Future distribution of cesium in the Tokyo Bay area}

From these results, it is possible to estimate the future distribution of cesium. In both rivers, cesium was deposited adhering to the fine-grained sediment near the mouths of the river. Though the exact position may vary, cesium is being buried by the much less contaminated sediment in both rivers. Also, since the dissolution of cesium from the bedload is minimal, the possibility of contaminating the seawater of Tokyo Bay is not low. Cesium is likely to be more 
deeply buried in the future, and the possibility for cesium to spread from Tokyo Bay to the Pacific Ocean is extremely low.

\section{Summary}

The fine-grained sediment flowing into Tokyo Bay was tracked using cesium as tracer. It was confirmed that the fine-grained sediment aggregated and was deposited at the estuaries. The results varied with the land use in the watershed area, as well as the geography and river flow. In the Edogawa River, the deposition peak was $10 \mathrm{~km}$ from the river mouth. In contrast, in the Arakawa River, cesium was transported as bedload with a speed of $3.5 \mathrm{~km} /$ year and was deposited ca. $3 \mathrm{~km}$ downstream from the river mouth. The effectiveness of cesium as a tracer of fine-grained sediment was confirmed, although the movement of the fine-grained sediment is still required to be tracked. It became clear that most of the fine-grained sediment did not move from the river mouth and is not likely to go into the Pacific Ocean.

\section{Acknowledgement}

I acknowledge financial support from the JST, the CREST program, "A Tracer Simulator of Fallout Radionuclides for Safe and Sustainable Water Use" of the Ministry of Education, Culture, Sports, Science and Technology (Japan).

\section{References}

[1] Reid, I. and J. B. Laronne, Bed Load Sediment Transport in an Ephemeral Stream and a Comparison with Seasonal and Perennial Counterparts, Water Resour. Res., 31(3), 773-781, 1995.

[2] Hirose, K, 2011 Fukushima Dai-ichi nuclear power plant accident: summary of regional radioactive deposition monitoring results, Journal of Environmental Radioactivity, Environmental Impacts of the Fukushima Accident (Part I), Vol. 111, 13-17, 2012.

[3] Chino, M., Nakayama, H., Nagai, H., Terada, H., Katata, G. and Yamazawa, H., Preliminary Estimation of Release Amounts of 131I and 137Cs Accidentally Discharged from the Fukushima Daiichi Nuclear Power Plant into the Atmosphere, Journal of Nuclear Science and Technology, Vol. 48, No. 7, p.1129-1134, 2011.

[4] Stohl. A., et al. Xenon-133 and caesium-137 releases into the atmosphere from the Fukushima Dai-ichi nuclear power plant: determination of the source term, atmospheric dispersion, and deposition, Atmospheric Chemistry and Physics Discussions, Vol. 11, pp. 28319-28394, 2011.

[5] Morino, Y., Ohara, T. and Nishizawa, M.: Atmospheric behavior, deposition, and budget of radioactive materials from the Fukushima Daiichi nuclear power plant in March 2011, Geophysical Research Letters, Vol. 38, L00G11, doi:10.1029/2011GL048689, 2011. 
[6] Yasunari, T. J., Stohl, A., Hayano, R. S., Burkhart, J. F., Eckhardt, S. and Yasunari, T.: Cesium-137 deposition and contamination of Japanese soils due to the Fukushima nuclear accident, Proceedings of the National Academy of Sciences of the United States of America, Vol. 108, No. 49, pp. 19530-19534, 2011.

[7] He, Q., D.E. Walling, Interpreting particle size effects in the adsorption of $137 \mathrm{Cs}$ and unsupported $210 \mathrm{~Pb}$ by mineral soils and sediments, Journal of Environmental Radioactivity, Volume 30, Issue 2, 117-137, 1996.

[8] Furukawa, K. and T. Okada, Tokyo Bay: Its Environmental Status - Past, Present, and Future, The Environment in Asia Pacific Harbours, Springer, pp. 15-34, 2006.

[9] Fujita M., Y. Koibuchi, Udin Hasanudinand K. Fujie: Microbial Response to Benthic Environment in Trench and Flat Seabed Sediments in Tokyo Bay, Japan, Journal of Coastal Research, 24 (1A), pp. 146-150, 2008.

[10] Koibuchi, Y., M. Isobe, Phytoplankton Bloom Mechanism in an Area Affected by Eutrophication: Tokyo Bay in Spring 1999, Coastal Engineering Journal, 49, 4, pp. 461-479, 2007.

[11] Japanese Ministry of Education, Culture, Sports, Science, and Technology (MEXT), Readings of environmental radiation level of dust and soil by monitoring in schools in Fukushima Prefecture. Available at: http://www.mext.go.jp/english/incident/1305657.htm., 2011.

[12] Japan Atomic Energy Agency, A trial calculation on total amount of radiation exposure during 2 months after the accident of Fukushima Daiichi Nuclear Power Plant in TEPCO. Available at: http://www.jaea.go.jp/jishin /kaisetsu03/kaisetsu03.htm (in Japanese), 2011.

[13] Nakagawa, Y., Nadaoka, K., Yagi, H., Ariji, R., Yoneyama, H., Shirai, K. Journal of JSCE, Ser. B2 (Coastal Engineering), Vol. 68, pp. 461-465, 2012. 